\title{
¿Por qué no se realiza actividad físico-deportiva en el tiempo libre? Motivos y correlatos sociodemográficos Why it does not make physical sport activity along spare time?
Reasons and sociodemographics correlations
}

\author{
Alfonso Valero Valenzuela*, Manuel Gómez López*, Juan Gavala González**, \\ Francisco Ruiz Juan* y María Elena García Montes* \\ * Universidad de Almería (España) y ** Universidad Pablo de Olavidades (España)
}

\begin{abstract}
Resumen: Se aborda los motivos aludidos por la población para no haber realizado nunca actividad físico-deportiva durante su tiempo libre, seleccionando una muestra de 1.116 sujetos y contemplando las variables sociodemográficas municipios por número de habitantes, sexo, edad, nivel de estudios y actividad principal. De los resultados obtenidos, destaca como principales motivos la falta de tiempo, porque no les gusta, y la salud. Los sectores de la población que dicen nunca haber realizado actividades físico-deportivas por falta de tiempo son quienes tienen de 30 a 59 años, personas con estudios de primaria o universitarios de grado medio y superior, trabajadores del sector público o privado y las amas de casa. Porque no les gusta la actividad físico-deportiva son los que tienen entre 15 y 29 años y los mayores de 59, los que acreditan tener estudios secundarios, de formación profesional, universitarios de grado medio, estudiantes, jubilados, pensionistas y rentistas, trabajadores del sector privado y parados. Por último, la salud influye especialmente en los habitantes mayores de 59 años, en los que no tienen estudios y en las amas de casa, jubilados, pensionistas y rentistas. Palabras clave: Motivos de práctica, tiempo libre, actividad físico-deportiva.
\end{abstract}

\begin{abstract}
The purpose of this paper is study the reasons mentioned by the population who have never made physical-sport activity during their free time, choosing a sample of 1,116 subjects and considering socio-demographics, municipalities by inhabitants number, sex, age, level of studies and main activity variables. Of the obtained results, it highlights lack for time, because they do not like, and the health. The population sectors that never say to have made physical-sport activity by lack of time are inhabitants between 30 and 59 years, people with primary, graduate and bachelor university studies, public or private workers and housewives. Because they do not like the physical-sport activity are people between 15 and 29 years and older than 59, those with high school, technical school and college studies, students, pensioners, retired and rentier people and public and private workers. Finally, health specially influences in those who are older than 59 years, those who haven't any studies, housewives and pensioners, retired and rentier people.
\end{abstract}

Key words: practice reasons, spare time, physical-sport activity.

\section{Introducción.}

La actividad físico-deportiva está ocupando, paulatinamente, ün lugar privilegiado dentro del marco de la sociedad actual, por un lado, debido a su poderosa capacidad educativa a lo largo de todas las etapas de vida y, por otro lado, por la enorme influencia positiva que tiene en la mejora de la calidad de vida de las personas (Sanz y Ponce, 2006). La magnitud social del fenómeno ha calado profundamente en virtud de la importante difusión que de él se hace en los diversos medios de comunicación, apareciendo un creciente interés por el desarrollo de los aspectos relativos al conocimiento y comprensión de los factores que determinan que las personas adquieran estilos de vida activos y perdurables en el tiempo (Gutiérrez Sanmartín, 1995; García Ferrando, 2001). En este sentido, e interesándonos el trabajar la motivación a partir de la elaboración de marcos teóricos que permitan ir más allá que la mera descripción (Castillo, Balaguer y Duda, 2000), el estudio de la motivación ha sido aplicado a diversos ámbitos de la vida cotidiana o de la conducta, siendo uno de ellos el de la actividad física y el deporte (Cervelló, Jiménez, Santos-Rosa y García, 2005).

Examinado las numerosas líneas de investigación desarrolladas entorno al tema de los motivos de práctica, abandono y no realización de la actividad físico-deportiva, encontramos que una gran variedad de autores como Weinberg y Gould (1996), Brownson et al. (2001); García Bengoechea y Spence (2002), Parks, Housemann y Brownson (2003), Nuviala et al. (2006), Díaz Suárez (2007), Ruiz Juan, García Montes y Díaz Suárez (2007), coinciden en apuntar como la razón más frecuente para la inactividad de práctica deportiva la falta de tiempo.

Fecha de recepción: 28-05-07 - Fecha de aceptación: 30-06-07 Correspondencia: Alfonso Valero Valenzuela

$\mathrm{C} /$ Santo Domingo Savio, $12,3^{\circ} \mathrm{F}$

23400 Úbeda - Jaén

E-mail: avalero@um.es
Cuando hablamos de motivación, nos referimos a sus tres dimensiones, la dirección-razones que llevan al sujeto a escoger o evitar una determinada actividad-, la intensidad -el mayor o menor esfuerzo que el sujeto emplea en la actividad- y la duración -tiempo que puede mantener el interés y el esfuerzo- (Castro, Piéron y González, 2006).

En España, los estudios a nivel nacional de García Ferrando (2001), sobre los motivos por los que no hace deporte la población señalan la falta de disponibilidad de tiempo libre como el principal motivo por el que no se hace deporte. Así, trabajos a nivel regional como los de Cañellas y Rovira (1995), en la población adulta barcelonesa aluden a causas ligadas con la falta de tiempo, al cansancio y a la falta de interés.

A nivel andaluz (Otero, 2004), los motivos para no practicar actividades físico-deportivas siguen siendo la falta de tiempo, la pereza y desgana y la edad. En Almería (Hernández Rodríguez, 2001) en un estudio realizado con la comunidad universitaria vuelve a encontrar la falta de tiempo, seguida de la pereza y desgana y salir muy cansado del trabajo o del estudio como los principales motivos aducidos por los no practicantes.

Conociendo cuáles son las principales causas por los que los ciudadanos no practican ningún tipo de práctica físico-deportiva, puede resultar interesante estudiar si existe algún tipo de variables sociodemográficas que nos indiquen cuáles son las poblaciones con mayor riesgo. De este modo, para comprender los comportamientos y, por consiguiente, las formas de promover la actividad física, los investigadores han estudiado los factores determinantes o correlatos que permiten establecer una asociación entre este tipo de práctica y su manifestación conductual (Dishman y Sallis, 1994; Sallis, 1999; Eyler, 2002), siendo considerados la educación, los ingresos económicos, el sexo y la edad en sentido negativo, como consistentes y poderosos determinantes de los comportamientos físico-deportivos.

En función del sexo, según el estudio realizado sobre la población cubana (Ruiz Juan, García Montes y Pérez, 2005), son las mujeres más propensas que los hombres a sentirse faltas de energía o destreza, coincidiendo con los resultados del estudio a nivel andaluz de Otero 


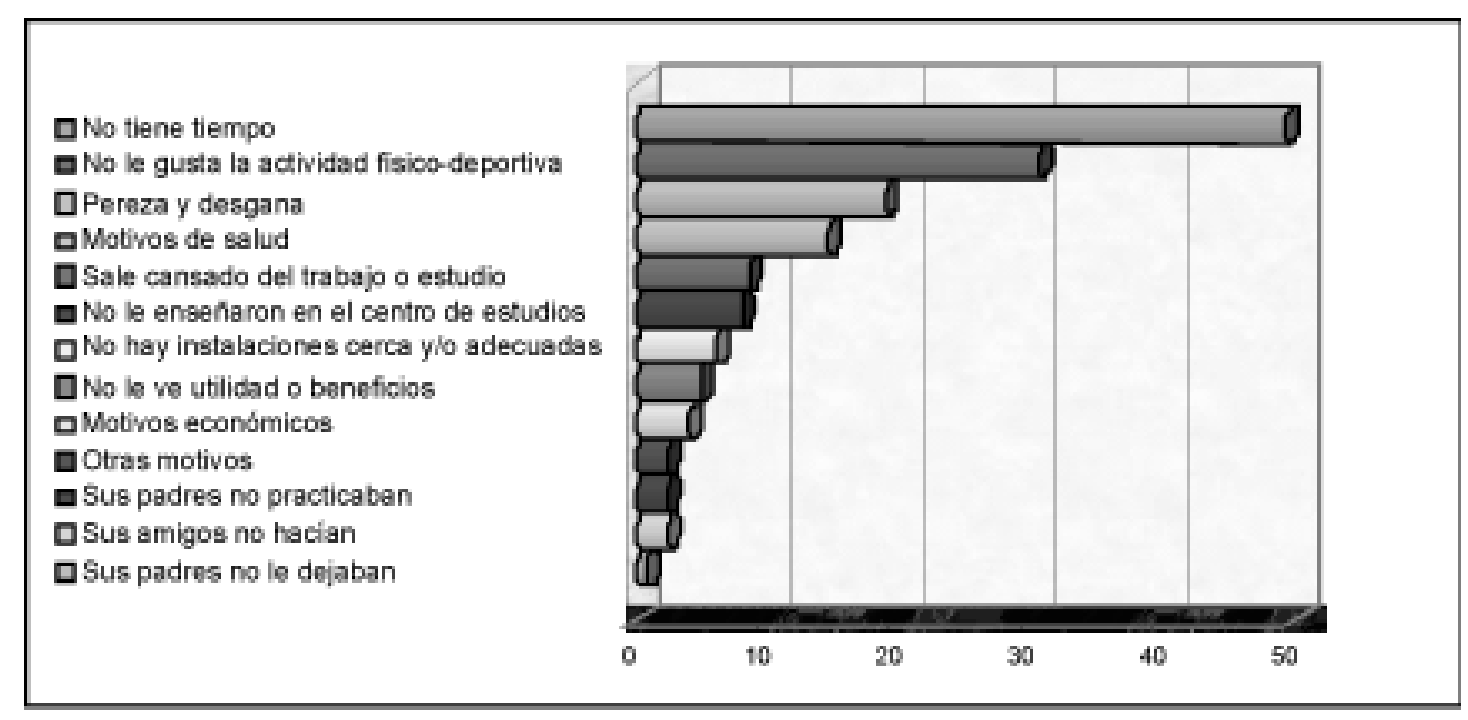

Figura 1. Motivos aludidos para no haber realizado nunca actividad físico-deportiva en su tiempo libre.

(2004), donde el tanto por ciento de mujeres que no practican actividad físico-deportiva por falta de tiempo es mayor frente a los hombres.

En cuanto a la edad, siguiendo el estudio a nivel nacional de García Ferrando (2001), los motivos aludidos por los jóvenes son porque no les gusta el deporte, así como la pereza y la desgana, mientras que los mayores aluden a motivos de salud (Ruiz Juan et al., 2005). Igualmente, las investigaciones revelan que el nivel de estudios se correlaciona con el nivel de práctica de una manera absoluta, y una situación similar se presenta con los ingresos económicos, dónde se manifiestan una correlación favorable. Porúltimo, las circunstancias son diferentes cuando de la ocupación laboral se trata, pues la relación no está muy clara, habiendo estudios que establecen asociación y otros que no lo hacen (Dishman y Sallis, 1994; Eyler, 2002; Parks et al., 2003).

El principal objetivo de este estudio es describir los motivos aludidos por los almerienses de la provincia de Almería, para no haber realizado nunca actividad físico-deportiva durante su tiempo libre, en función de una serie de variables sociodemográficas como son los municipios por número de habitantes, sexo, nivel de estudios y actividad principal.

\section{Material y método.}

Comenzando por la descripción de la población y la muestra, esta investigación se hallevado a cabo en la provincia deAlmería, escogiéndose para ella todos los almerienses mayores de 14 años. Los datos fueron facilitados por el INE (Instituto Nacional deEstadística) correspondientes a la renovación del padrón de la provincia de Almería del año 1.999.

Éstos, se distribuyen por estratos, atendiendo al número de habitantes por municipio (más de 50.000 habitantes, de 20.001 a 50.000 habitantes, de 10.001 a 20.000 habitantes, de 5.001 a 10.000 habitantes, de 2.001 a 5.000 habitantes y menos de 2.001 habitantes), a los municipios, distritos, secciones censales (determinados en los tres casos para su identificación, según codificación numérica) y sexo (varones y mujeres). Igualmente, se nos proporcionó la distribución de la población por sexo, municipios y edad (grupos quinquenales).

A partir de estos datos, extraemos que la población censada en la provincia de Almería, según el padrón del año 99, asciende a 512.843 habitantes y que la cifra de almerienses mayores de 14 años es de 416.973 habitantes; de los cuales, 207.013 son varones y 209.960, mujeres.

Teniendo en cuenta esto, en el caso de la población general (416.973 habitantes censados en la provincia de Almería mayores de 14 años), el tamaño de la muestra se estimó en 1.116 sujetos. Esto supone una validez en los resultados con un margen de error muestral del $\pm 3 \%$ y un nivel de confianza del $95.5 \%$. El procedimiento de muestreo utilizado ha sido polietápico con afijación proporcional, recurriendo a dos tipos de muestreo: el muestreo aleatorio estratificado y el muestreo aleatorio por conglomerados.

Como nuestra investigación se basa en el estudio y análisis de variables subjetivas de un amplio número de individuos tales como opiniones, valoraciones, pensamientos, comportamientos, intereses y motivaciones para luego extrapolar los resultados al total de la población, es la encuesta la técnica más apropiada y el cuestionario, el instrumento a utilizar. Ha sido el propio entrevistador el que ha tomado nota de las respuestas que daban las personas entrevistadas, realizándose éstas en la propia vivienda del encuestado. El trabajo de campo se llevó a cabo durante los meses de febrero y marzo de 2002 aplicando el sistema de rutas aleatorias por secciones censales, lo que permitió seleccionar aleatoriamente la muestra dentro de cada sección censal.

Para obtener la información necesaria en esta investigación se ha utilizado el cuestionario denominado «Hábitos físico-deportivos y estilos de vida» (Ruiz Juan y García Montes, 2005). Los bloques temáticos del cuestionario incluidos en esta investigación son: disponibilidad y ocupación del tiempo libre, valoración de la educación física recibida, hábitos físico-deportivos de tiempo libre (interés, práctica y demanda de actividades físico-deportivas), percepción de la condición física y del estado de salud, enfermedades más habituales y hábitos de consumo (alimentación, medicamentos, alcohol, tabaco, otras drogas). Se le han pasado las correspondientes pruebas de validez de contenido y de constructo. Igualmente, reseñar que hemos constatado la fiabilidad del cuestionario, mediante los diferentes estudios piloto, así como por las diferentes aportaciones hechas por los expertos que han colaborado en la investigación.

\section{Resultados y discusión.}

Debido a que nuestra investigación se centra en aquellos sujetos que nunca han practicado actividad físico-deportiva, del $100 \%$ de la muestra se han eliminado aquellos que si practican y los que la han abandonado (738 sujetos), quedándonos con una muestra para este estudio de 378 sujetos, los cuales representan casi el treinta y cuatro por ciento del total dela población.

Centrándonos en los motivos que ha aludido lapoblación almeriense que nunca ha practicado actividad físico-deportiva, hallamos que casi la mitad de la población aduce a la escasez de tiempo. La segunda razón más numerosa es porque no le gusta la actividad físico-deportiva, con treinta puntos y, con algo más de un dieciocho por cierto, están los que dicen no haber practicado por pereza y desgana. El siguiente motivo más aludido son los problemas de salud, con casi quince puntos porcentuales, y el resto de razones no llega a sobrepasar los diez puntos porcentuales (figura 1). 
Tabla 1. Motivos por los que los almerienses no han realizado nunca actividad físico-deportiva durante su tiempo libre. Frecuencia y porcentaje por el orden de los motivos aludidos.

\begin{tabular}{|c|c|c|c|c|c|c|}
\hline & \multicolumn{2}{|c|}{$1^{\circ}$ motivo } & \multicolumn{2}{|c|}{$2^{\circ}$ motivo } & \multicolumn{2}{|c|}{$3^{\circ}$ motive } \\
\hline & $\mathrm{N}$ & $\%$ & $\mathrm{~N}$ & $\%$ & $\mathrm{~N}$ & $\%$ \\
\hline Porque no le gusta la actividad fisico-deportiva. & 88 & 23.3 & 16 & 4.2 & 4 & 1.1 \\
\hline Por motivos de salud. & 30 & 7.9 & 16 & 4.2 & 5 & 1.3 \\
\hline Porque no le enseñaron en el centro de estudios. & 12 & 3.2 & 5 & 1.3 & 6 & 1.6 \\
\hline Porque no k ve utilidad/Nole ve beneficios. & 6 & 1.6 & 6 & 1.6 & 1 & 0.3 \\
\hline Porque no tiene tiempo. & 129 & 34.1 & 31 & 8.2 & 5 & 1.3 \\
\hline Porque no hay instalaciones cerca y $/ 0$ adecuadas & 8 & 2.1 & 11 & 29 & 3 & 0.8 \\
\hline Porqwe sale muy carsado del trahajo o del estudio. & 10 & 2,6 & 15 & 4.0 & 4 & 1.1 \\
\hline Por percza y desgana. & 17 & 4.5 & 35 & 9.3 & 15 & 4.0 \\
\hline Por motivos económicos & 6 & 1.6 & 6 & 1.6 & 3 & 0.8 \\
\hline Porque sus amigos no hacian. & 0 & 0.0 & 3 & 0.8 & 1 & 0.3 \\
\hline Porque sus padres no practicakun. & 3 & 0,8 & 3 & 08 & 4 & 1.1 \\
\hline Porque sus padres no le dejaban. & 1 & 0.3 & 2 & $0 s$ & 5 & 13 \\
\hline Otras motivos: & 12 & 3.2 & 16 & 4.2 & 6 & 1.6 \\
\hline $\mathrm{NS} / \mathrm{NC}$ & 56 & 14.8 & 229 & 60.6 & 331 & 87.6 \\
\hline
\end{tabular}

Estos resultados están en sintonía con los obtenidos por García Ferrando (2001), apareciendo la falta de tiempo como el primer motivo que esgrime la población española con un $45 \%$ para justificar la no realización de actividad físico-deportiva, seguido de la falta de interés y gusto por el deporte (39\%). Teniendo en cuenta los resultados obtenidos por Cañellas y Rovira (1995) y por Otero (2004), otros motivos, además de la falta de tiempo, también son aludidos por los ciudadanos de diferentes regiones, como la catalana y la andaluza que apuntan a la falta de interés y a la pereza o desgana, estando en la misma línea de nuestro estudio realizado con los almerienses.

En cuanto al orden de los motivos de por qué los almerienses nunca han realizado actividad físico-deportiva, llama poderosamente la atención la gran cantidad de personas que dice no saber o no contestar en el segundo y el tercer motivo, con más de un sesenta y casi un noventa por

Tabla 2. Motivos por los que los almerienses no han realizado nunca actividad físico-deportiva durante su tiempo libre. Porcentaje y distribución por municipios.

\begin{tabular}{ccc}
\hline \hline & $\begin{array}{c}\text { Perezy } \\
\text { dagans }\end{array}$ & Total \\
\hline Mas de 50.000 & 40.8 & 43.7 \\
De 20,001 a 50,000 & 12.7 & 10.3 \\
De 10.001 a 20.000 & 23.9 & 14.0 \\
De 5.001 a 10.000 & 4.2 & 11.1 \\
De 2.001 a 5.000 & 12.7 & 12.7 \\
Menos de 2.000 & 5.6 & 8.2 \\
\hline$X^{2}-11.201$ & p-valor $=0.048$ \\
\hline \hline
\end{tabular}

ciento respectivamente(tabla 1). En cuanto al primer motivo, la mayoría suele poner como excusa la falta de tiempo (34.1\%), o porque no le gusta la actividad físico-deportiva (23.3\%), o por problemas de salud (7.9\%), siendo el resto de porcentajes muy pequeños, no superando el cinco por ciento (tabla 1 ).

Analizando los diversos motivos por los que nunca han realizado actividad físico-deportiva los almerienses, en función de los municipios según el número de habitantes, encontramos que únicamente se hallan diferencias estadísticamente significativas para el motivo de «pereza y desgana» ( $<<0.05)$, siendo los municipios entre 10.001 y 50.000 habitantes quienes más aluden a esta causa (tabla 2). Por lo tanto, el lugar dónde reside un sujeto no influye en el resto de los motivos aludidos para no haber realizado nunca actividad físico-deportiva en el tiempo libre.

Por lo que respecta a la variable sexo, tenemos que indicar que no se han encontrado diferencias estadísticamente significativas en ninguno de los motivos aludidos. Esto nos lleva a afirmar que el sexo no influye en esta población objeto de estudio, es decir, que tanto hombres como mujeres manifiestan los mismos motivos por los que nunca realizan actividad físico-deportiva en el tiempo libre. Adiferencia de los resultados aquí hallados, otras investigaciones como la de Ispizua (1993), García Montes, Rebollo, Martínez y Oña (1996), García Montes (2001), García Montes, Hernández, Oña, Godoy y Rebollo (2001), Otero (2004) y Nuviala Nuviala, Ruiz Juan, García Montes (2003), sí obtienen diferentes motivos para hombres y mujeres, de tal modo que, las mujeres aluden a la falta de tiempo, mientras que los varones manifiestan sobre todo razones relativas a las repercusiones que derivan del incremento de edad y de los motivos de la salud.

Tabla 3. Motivos por los que los almerienses no han realizado nunca actividad físico-deportiva durante su tiempo libre. Porcentaje y distribución por grupos de edad.

\begin{tabular}{|c|c|c|c|c|c|}
\hline & $\begin{array}{l}\text { No dispone de } \\
\text { tiempe }\end{array}$ & $\begin{array}{c}\text { No le gusta } 12 \\
\text { actividad }\end{array}$ & Salud & $\begin{array}{c}\text { Porques no le } \\
\text { cnseñason en } \\
\text { el ceatro de } \\
\text { cstudios }\end{array}$ & Total \\
\hline De 15 a 29 años & 11.8 & 183 & 3.6 & 10.0 & 12.4 \\
\hline De 30 ล 44 años & 31.7 & 21.7 & 7.3 & 0.0 & 23.8 \\
\hline De 45 a 59 años & 32.3 & 18.3 & 182 & 46.7 & 24,6 \\
\hline De 60 años en adelan to & 24.2 & 41.7 & 70.9 & 43.3 & 39.2 \\
\hline$X^{2}=$ & 39.386 & 7.796 & 29056 & 14.507 & \\
\hline p-valor $=$ & 0,000 & 0.049 & 0.000 & 0.002 & \\
\hline
\end{tabular}


Tabla 4. Motivos por los que los almerienses no han realizado nunca actividad físico-deportiva durante tiempo libre. Porcentaje y distribución por nivel de estudios.

\begin{tabular}{|c|c|c|c|c|}
\hline & $\begin{array}{l}\text { No dispone de } \\
\text { ti anpo }\end{array}$ & $\begin{array}{c}\text { No le gusts la } \\
\text { actividad }\end{array}$ & Salud & Total \\
\hline Sin estudios & 30.3 & 339 & 582 & 37.8 \\
\hline Primarios completos & 50.3 & 365 & 273 & 42.3 \\
\hline Formación Profesional & 7.0 & 122 & 73 & 7.4 \\
\hline Secundarios: BUP. COU & 59 & 113 & 36 & 7.7 \\
\hline Universi tarics de grado medio & 3.8 & 52 & 1.8 & 3.2 \\
\hline Universitarios de grado superior & 27 & 0.9 & 18 & 1.6 \\
\hline$x^{2}=$ & 39,386 & 12.374 & 12336 & \\
\hline p-yakt $=$ & 0.008 & 0.030 & 0.030 & \\
\hline
\end{tabular}

Si analizamos la población almeriense por grupos de edad, encontramos diferencias significativas para los motivos de falta de tiempo, falta de gusto por la actividad, cuestiones relacionadas con la salud y porque no le enseñaron en su centro de estudios. La falta de tiempo es el motivo aludido por las poblaciones entre 30 y 59 años ( $p<0.001)$, mientras que son los jóvenes de 15 a 29 años y los mayores de 60 años quienes más afirman no practicar porque no les gusta ( $<<0.05)$, y la salud es la principal causa manifestada por los adultos mayores de 59 años ( $\mathrm{p}<0.001)$.Además, es de destacarcomo la enseñanza que recibieron los sujetos en sus centros de estudio, es la causa expuesta por los ciudadanos con más de 44 años $(\mathrm{p}<0.005)$, cuestión que afortunadamente no se recoge en generaciones más jóvenes y que, por tanto, consideramos ha sido subsanada por los centros educativos con el paso de los años (tabla 3).

García Ferrando (2001), en su estudio a nivel nacional, coincide en parte con los datos aquí arrojados, al obtener que los más jóvenes reconocen en mayor proporción quenoles gusta el deporte, al igual que ocurre entre los jóvenes andaluces (Otero, 2004). La población entre los 30 y 60 años son quienes más reconocen no practicar por falta de tiempo y son los adultos-mayores quienes aluden a otros motivos como la salud (Ruiz Juan et al., 2005), o la edad (Otero, 2004).

En cuanto a la variable sociodemográfica nivel de estudios, encontramos denuevo diferencias significativas para varios delos motivos aludidos por los almerienses, como es la falta de tiempo, problemas de salud y falta de gusto por la actividad. En concreto, la ausencia de tiempo disponible es la causa enunciada por los sujetos que tienen la primaria completa y estudios universitarios de grado medio y superior $(\mathrm{p}<0.01)$. Otro de los principales motivos por los que no se practica actividad físico-deportiva durante el tiempo libre, es la falta de gusto por el ejercicio, siendo la causa mencionada por los sujetos que tienen la formación profesional acabada, estudios secundarios y los universitarios de grado medio $(p<0.05)$. Finalmente, la salud, es la causa que alegan las personas que no tienen estudios $(\mathrm{p}<0.05)$ (tabla 4$)$.

A diferencia de otros factores, tras el análisis realizado a esta variable sociodemográfica, no hemos encontramos un patrón de conducta que pueda estar en consonancia con los estudios de Dishman y Sallis (1994), Sallis (1999) y Eyler (2002), que mantienen que existe una relación absoluta entre el nivel de estudios y la práctica de actividad físicodeportiva. No obstante, consideramos que es conveniente realizar más estudios en la misma línea que traten de arrojar datos más concluyentes al respecto.

Por último, se han vuelto a detectar diferencias significativas para varios de los motivos en función de la actividad principal. Los motivos relacionados con la falta de tiempo son aducidos por las amas de casa y los trabajadores del sector público y privado $(\mathrm{p}<0.001)$. Debido a que no les gusta la actividad, es el motivo mencionado por todos los sectores de la población, excepto las amas de casa y los trabajadores del sector público $(\mathrm{p}<0.05)$, mientras que el grupo más numeroso de las amas de casa, jubilados, pensionistas y rentistas suelen decir que no lo hacen por motivos de salud $(p<0.001)$. En cambio, salir cansado del trabajo o del estudio son las causas alegadas por los estudiantes y los trabajadores del sector público y privado $(\mathrm{p}<0.005)$, lo cual, por otra parte también es lógico, tal y como está descrito este factor (tabla 5).

A ráz de los resultados arrojados por el análisis estadísitico correlacional entre los motivos aludidos por los sujetos que nunca han practicado actividad físico deportiva y la actividad principal, los motivos aludidos por los diferentes sectores están en relación con sus circunstancias laborales, de tal modo, que difícilmente un parado puede alegar como motivo falta de tiempo, y por otro lado, es lógico que las personas de mayor edad, aludan a motivos de salud como principal causa de su falta de actividad física. Finalmente, los trabajadores son quienes pueden esgrimir como excusa la falta de tiempo, que le provoca su empleo o salir cansado del mismo.

\section{Conclusiones.}

Cuando se les pregunta a los almerienses por los motivos que les llevan a no haber practicado nunca actividad físico-deportiva, obtenemos que básicamente son porque no tienen tiempo, porque no les gusta o por motivos de salud. Los sectores de la población que dicen no haber

Tabla 5. Motivos por los que los almerienses no han realizado nunca actividad físico-deportiva durante tiempo libre. Porcentaje y distribución por actividad principal.

\begin{tabular}{|c|c|c|c|c|c|}
\hline & $\begin{array}{l}\text { No dispone de } \\
\text { tiempo }\end{array}$ & $\begin{array}{l}\text { No le gusta la } \\
\text { actividad }\end{array}$ & S slual & $\begin{array}{l}\text { Sale cansado } \\
\text { Ad trabajo o } \\
\text { estadio }\end{array}$ & Total \\
\hline Ama de casa & 28.5 & 200 & 36.4 & 12.5 & 27.9 \\
\hline Estudiante & 1.1 & 7.0 & 00 & 12.5 & 4.2 \\
\hline Jabilado'pension ista' rentista & 16.7 & 330 & 49.1 & 15.6 & 29.2 \\
\hline Prado & 2.2 & 6.1 & 36 & 3.1 & $3, A$ \\
\hline Trabaja en sector pubblico & 12.9 & 6.1 & 9.1 & 25.0 & 9.5 \\
\hline Trabaja en seckor priv ado & 38.7 & 27.8 & 1.8 & 313 & 25.7 \\
\hline$x^{3}=$ & 58.595 & 13.080 & 27.490 & 19.943 & \\
\hline $\mathrm{p}-\mathrm{valor}=$ & 0.000 & 0.023 & 0.000 & 0.001 & \\
\hline
\end{tabular}


practicado actividad físico-deportiva por el primero de los motivos, es decir, porque no tienen tiempo son los ciudadanos de 30 a 59 años, los que tienen la primaria completa o estudios universitarios de grado medio o superior, trabajadores del sector privado y público y las amas de casa. Por otro lado, el segundo motivo aludido, referido a que no les gusta, es mencionado por los jóvenes de 15 a 29 años y los mayores de 60, los que tienen estudios secundarios, de formación profesional, universitarios de grado medio, estudiantes, jubilados, pensionistas y rentistas, trabajadores del sector privado y parados. Así mismo, los ciudadanos que confiesan no haber practicado actividad físico-deportiva por problemas de salud son, sobre todo, los sujetos de más de 59 años, los que no tienen estudios, las amas de casa y los jubilados, pensionistas y rentistas. Para terminar, cabe resaltar que a diferencia de otras investigaciones, en el caso concreto de este estudio, el sexo no influye en los motivos por los que nunca se realiza actividad físico-deportiva en el tiempo libre, es decir, que tanto hombres como mujeres manifiestan los mismos motivos.

\section{Bibliografía.}

Brownson, R. C., Baker, E. A., Housemann, R. A., Brennan, L. K. y Bacak, S. J. (2001). Environmental and policy determinants of physical activity in the United States. American Journal of Public Health, 91-1995-2003.

Cañellas, A. y Rovira, J.(1995). Los hábitos deportivos de la población adulta barcelonesa (15 a 59 años). Apunts: Educación Física y Deportes, 42, 75-79.

Castillo, I., Balaguer, I. y Duda, J. L. (2000). Las orientaciones de meta y los motivos de práctica deportiva en los jóvenes deportistas valencianos escolarizados. Revista de Psicología del Deporte, 9, 37-50.

Castro, M. J., Piéron, M. y González, M. A. (2006). Actitudes y motivación en Educación Física escolar. Retos. Nuevas tendencias en Educación Física, Deporte y Recreación, 10, 5-22.

Cervelló, E. M., Jiménez, R., Santos-Rosa, F. J., García, T. (2005). El clima motivacional en las clases de Educación Física: una aproximación práctica desde la Teoría de Metas de Logro.Apunts 81, 21-28.

Díaz Suárez, A. (2007). La Educación Física y el Deporte Escolar en la Región de Murcia. RETOS. Nuevas tendencias en Educación Física, Deporte y Recreación, 11, 26-32.

Dishman, R. K. y Sallis, J. F. (1994). Determinants and interventions for physical activity and exercise. En C. Bouchard, R. J. Shephard, y T. Stephens (Eds.), Physical activity, fitness, and health: International proceeding and consensus statement (pp. 214-238). Champaign, IL: Human Kinetics.

Eyler, A. A. (2002). Correlates of physical activity: Who's active and who's not?. Arthritis Care and Research, 49(1), 136-140.

García Bengoechea, E. y Spence, J.C.(2002). Alberta Surveyon Physical Activity. Edmonton: Centre for Active Living.

García Ferrando, M. (2001). Los españoles y el deporte: prácticas y comportamientos en la última década del siglo XX. Encuesta sobre hábitos deportivos de españoles, 2000. Madrid: Ministerio de Educación, Cultura y Deporte. Consejo Superior Deportes.

García Montes, M. E. (2001). Actitudes y comportamientos de la mujer granadina ante la práctica física de tiempo libre. Granada: Tesis doctoral, Universidad de Granada.

García Montes, M. E., Hernández, A. I., Oña, A., Godoy, J. F. y Rebollo, S. (2001). La práctica física de tiempo libre en las mujeres. Motricidad. Revista Euro-Americana de Ciencias de la Actividad Física y el Deporte, 7, 145-186.

García Montes, M. E., Rebollo, S., Martínez, M. y Oña, A. (1996) Estudio de hábitos deportivos en la provincia de Granada. Motricidad. Revista Euro-Americana de Ciencias de la Actividad Física yel Deporte, 2, 55-73.
Gutiérrez Sanmartín, M.(1995). Valores socialesy deporte: La actividad física y el Deporte como transmisores de valores sociales y personales. Madrid: Gymnos.

Hernández Rodríguez, A. I. (2001). Análisis de la demanda de la comunidad universitaria almeriense en actividades físicodeportivas. Estudio de adecuación de la oferta. Almería: Tesis Doctoral, Universidad de Almería.

Ispizua, M. (1993). Hábitos deportivos de la población de la margen izquierda. Baracaldo, Portugalete, Santurzi y Sestao.(1992). Bilbao: Ayuntamientos de Baracaldo, Portugalete, Santurtzi y Sestao. Eusko Jaurlatitza. Gobierno Vasco. Birkaiko Foru Aldundia. Diputación Foral de Bizkaia. EUDEL Y KAIT.

Nuviala Nuviala, A. Ruiz Juan, F. y García Montes, M. E. (2003). Tiempo libre, ocio y actividad física en los adolescentes. La influencia de los padres. RETOS. Nuevas tendencias en Educación Física, Deportey Recreación, 6, 13-20.

Nuviala Nuviala, A., Ruiz Juan, F., García Montes, M. E. y Díaz Suárez, A. (2006). Motivos de práctica y tasa de participación en actividades físico deportivas organizadas entre adolescentes que viven en dos entornos rurales. Cuadernos de Psicología del Deporte, 6, 69-80.

Otero, J. M. (2004). Hábitos y actitudes de los andaluces ante el deporte (2002). Cádiz: Consejería de Turismo y Deporte. Observatorio del Deporte Andaluz.

Parks, S. E, Housemann, R. A, y Brownson, R. C. (2003). Backgrounds in the United States and rural adults of various socioeconomic Differential. Journal Epidemiology Community Health, 57, 29-35

Ruiz Juan, F. y García Montes, M. E. (2005). Significado del estudio, objetivos y metodología. En: F. Ruiz Juan y M. E. García Montes (Eds.), Hábitos físico-deportivos de los almerienses en su tiempo libre (pp. 1-22).Almería: Servicio Publicaciones de la Universidad deAlmería.

Ruiz Juan, F., García Montes, M. E. y Díaz Suárez, A. (2007). Análisis de las motivaciones de práctica de actividad física y de abandono deportivo en la Ciudad de la Habana (Cuba). Anales de Psicología, 23, 152-166.

Ruiz Juan, F., García Montes, M. E. y Pérez Sánchez, A. (2005).

Estilos de vida en ciudad de la Habana-Cuba. Hábitos físicodeportivos y de salud. Madrid: Gymnos.

Sallis, J. F. (1999). Influences on Physical Activity of Children, Adolescents, and Adults. President's Council on Physical Fitness and Sport. Research Digest, 1(7), 1-4.

Sanz, E. y Ponce, A. (2006). La necesidad de educar la dimensión del ocio físico-deportivo. Propuesta surgida de un estudio centrado en una comunidad universitaria. Tandem, 20, 73-88.

Weinberg, R. y Gould, D. (1996). Fundamentos de psicología del deporte y el ejercicio físico. Barcelona: Ariel Psicología.
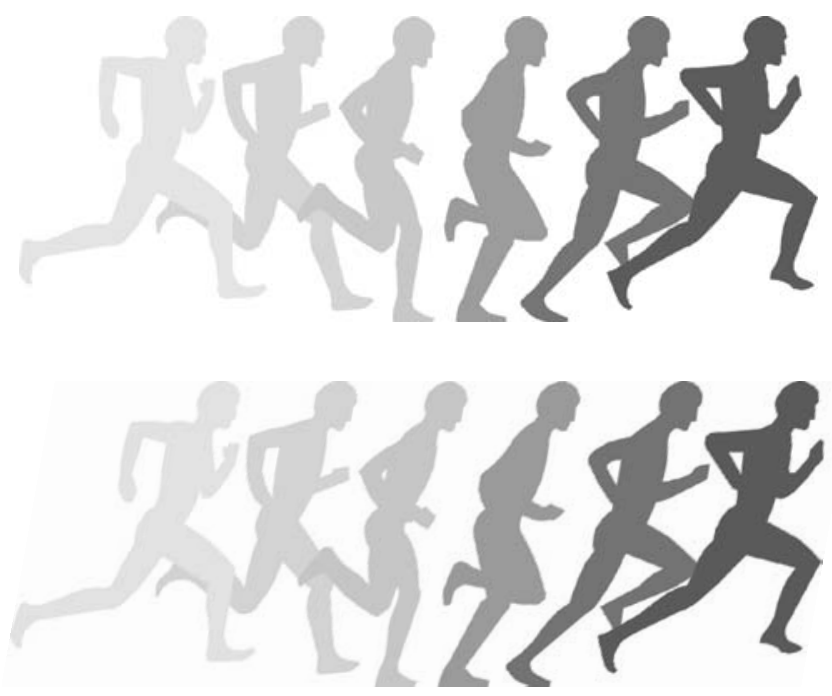